\title{
Pluralismo agonista en la internacionalización de los estudios latinoamericanos de la comunicación: reflexiones a partir de la práctica
}

\author{
Practice-based reflections on the internationalization of Latin American \\ communication studies from a pluralist-agonistic perspective
}

\section{Martín Becerra}

Universidad Nacional de Quilmes, Buenos Aires, Argentina aracabecerrađgmail.com https://orcid.org/00000-0002-8844-8664

\section{Florencia Enghel}

Universidad de Jönköping, Jönköping, Suecia

florencia.Engheldju.se

https://orcid.org/0000-0003-4760-754X

\section{Resumen}

Este artículo examina las prácticas de circulación de la producción teórica en el campo de estudios de la comunicación y los medios en la actual fase de internacionalización y revisa las relaciones problemáticas entre Sur y Norte. En base a la revisión crítica del proceso de edición de un número especial de una revista prestigiosa del campo disciplinar producida en inglés, reflexionamos acerca de los límites estructurales que influyen en la peculiar presencia y ausencia de producciones latinoamericanas e hilvanamos un conjunto de lecciones sobre los (des)encuentros entre el sistema de publicaciones científicas angloparlante y los estudios latinoamericanos y los desafíos que éstos implican para la internacionalización de los estudios de comunicación.

Palabras clave: América Latina, estudios en medios y comunicación, internacionalización, pluralismo, división Norte/Sur.

\begin{abstract}
This article examines the practices aimed at circulating theoretical work in the field of media and communication studies at the current stage of internationalization and reviews the problematical relationship between North and South in this respect. By scrutinizing the editorial process of a prestigious scholarly publication in the field, the article considers the structural limits shaping the particular presence or absence of Latin American contributions to media and communication studies. In doing so, this article discusses a set of lessons about (mis)matchings between the English-speaking system of scientific publications and Latin American studies, as well as the challenges such frictions imply for the internationalization of communication studies.
\end{abstract}

Keywords: Latin America, media and communication studies, internationalization, pluralism, North/South divide 


\section{Introducción}

La ausencia de contribuciones latinoamericanas al campo de los estudios sobre medios y comunicación en la literatura publicada en inglés fue reconocida por investigadores de Estados Unidos hace casi treinta años (Chaffee, Gomez-Palacio \& Rogers, 1990; Rich, 1993), pero el problema perdura. En años recientes, la cuestión de cómo incorporar a los estudios latinoamericanos de medios y comunicación a los círculos académicos de Estados Unidos y Europa ha sido objeto de discusión desde perspectivas normativas, pero raras veces encarada en la práctica (Waisbord, 2016).

En 2016, un estudio del archivo de la revista académica de alto impacto Communication Theory (CT), publicada por la Asociación Internacional de la Comunicación [International Communication Association, ICA] en busca de artículos que se refirieran a América Latina, nos permitió determinar que los mismos estaban seriamente subrepresentados en dicho archivo (Enghel $\&$ Becerra, 2018). A partir de ese hallazgo, en 2018 CT editó un número especial integrado exclusivamente por contribuciones latinoamericanas a los debates globales propios del campo. Atenta al desfase que existe entre la producción académica generada a nivel regional (creciente en cantidad y variedad desde fines de la década del ochental y su circulación internacional, la edición especial incluyó trabajos recientes y puso énfasis, así, en el presente como una dimensión temporal desatendida de la muchas veces predicada pero jamás alcanzada internacionalización del campo.

Este artículo reflexiona a partir de las lecciones aprendidas sobre cómo se vincula el sistema de publicaciones científicas angloparlante con los estudios latinoamericanos, tomando como base la experiencia de producir dicha edición especial de CT. Consideramos, además, de qué maneras América Latina podría contribuir al pluralismo internacional en este campo, teniendo en cuenta las limitaciones estructurales y el consecuente diferencial en la capacidad de acción de académicxs a uno y otro lado de la división Norte-Sur.

\section{Problema, objeto de estudio y conceptos teóricos centrales}

\subsection{La escasez de contribuciones de y sobre América Latina en las revistas académicas internacionales: ausencia de pluralismo}

En 2007, Sonia Livingstone, por entonces presidenta electa de ICA, planteó una pregunta que sigue vigente: "¿Qué significa internacionalizar los estudios de medios y comunicación?"1 (Livingstone, 2007, p. 273). En su análisis, Livinsgtone situaba el problema irresuelto de la internacionalización en "la intersección entre el campo de los medios y la comunicación y las múltiples y superpuestas asociaciones nacionales, regionales e internacionales que representan a las y los investigadoras/es individuales", y posicionaba, así, a dichas asociaciones como actores influyentes (p. 273). Citando una observación de Curran y Park según la cual se ha transformado en "rutina realizar observaciones universalistas sobre los medios en libros publicados en inglés a partir de evidencia derivada de un pequeño puñado de países" (Curran \& Park, 2000, p. 3), Livingstone llamaba la atención sobre "la gran diferencia entre el proyecto de internacionalización que busca expandir el alcance de un campo de estudios mayormente occidentalizado y aquel que busca, en cambio, diversificar o des-occidentalizar el campo" y explicaba que, si bien no le había resultado difícil acordar un conjunto de estándares de excelencia -calidad, originalidad, inclusión, diversidad, apertura, rigor y profesionalismo- para conducir a la ICA, “las prácticas requeridas para implementar estos principios [...] requieren esfuerzo, negociación y revisión crítica continuos" (Livingstone, 2007, p. 275). Según ella, "puede ser precisamente la brecha entre aspirar a una comunidad global de ideas compartidas y las realidades marcadas por formas de trabajar y pensar diferentes, o incluso en conflicto, lo que plantea las tareas más difíciles en pos de servir a los intereses de sus miembros" (p. 276; las itálicas son nuestras).

La tensión entre las diferentes maneras de entender qué significa en la práctica la internacionalización de los estudios de medios y comunicación, por un lado, y la distancia entre las distintas "formas de trabajar y pensar" que Sonia Livingstone identificara en 2007, por otro, constituyeron elementos cruciales de la experiencia editorial que analizamos 
en este artículo. ¿En qué consistió la experiencia? Habiendo constatado la escasez de contribuciones de y sobre América Latina en CT, le propusimos a su entonces editora publicar una edición especial de la revista dedicada a subsanar el problema. El llamado a contribuciones latinoamericanas recientes de relevancia para los debates globales, realizado en 2016, dio lugar dos años más tarde a la publicación de "La teoría latinoamericana de la comunicación hoy: cartografía de los desarrollos contemporáneos y su relevancia global" (Enghel \& Becerra, 2018). El proceso de producción de dicha edición especial nos obligó a definir dos conceptos medulares -latinoamericanidad y pluralismo- y a tomar decisiones constantes respecto de cómo operacionalizar estos conceptos a medida que nos vinculábamos con autorxs y evaluadorxs tanto del Sur como del Norte. Tal como lo señalara Livingstone (2007, p. 126), el "esfuerzo, [la] negociación y [la] revisión crítica" fueron necesarios a cada paso.

Las revistas publicadas por la ICA, CT incluida, están lejos de ser plurales en términos del país de origen y/o la afiliación institucional de los autores (Waisbord, 2019, pp. 95-99)². Desde su posición como ex editor de otra revista de la ICA, Journal of Communication, Waisbord documenta las "disparidades geográficas en las publicaciones académicas" Ip. 99) y argumenta que lo que él denomina la "globalización institucional" de la comunicación académica -es decir, su estructura organizacional tal como la definen universidades, asociaciones profesionales, publicaciones y conferencias- ha conducido a "la creciente presencia de estudios académicos producidos en un número relativamente pequeño de países" y a la notoria ausencia de gran parte de América del Sur (p. 98; ver también Ganter \& Ortega, 2019). Dicho de otro modo: en las publicaciones de la ICA escasean tanto la diversidad como el pluralismo y sólo unos pocos países además de EE. UU. están representados en ellas ${ }^{3}$.

\subsection{El pluralismo en las revistas académicas de alto impacto publicadas en inglés: hacia una definición}

Como bien argumentan Raeijmaekers y Maeseele (2015) en su análisis de las divergencias conceptuales y los supuestos normativos acerca de los vínculos entre medios, pluralismo y democracia, la noción de pluralismo merece un examen cuidadoso:

es notable hasta qué punto el pluralismo de los medios sirve como expresión de moda o como un concepto descontextualizado que se da por sentado. En general, no está claro qué se quiere decir al referirse a contenidos pluralistas o cómo debieran operar los medios pluralistas en las sociedades democráticas de Occidente (2015, p. 1043).

A nuestro modo de ver, esta falta de claridad es también característica de los debates sobre la internacionalización de las revistas académicas angloparlantes sobre medios y comunicación, y debe ser abordada.

Según Carles Llorens, lo que define al pluralismo es precisamente la suma de pluralidad y diversidad (2002, p. 125; Doyle, 1997; Napoli, 1999). Adoptamos aquí esa definición ${ }^{4}$ y la consideramos para analizar la problemática ausencia de contribuciones de América Latina en las revistas académicas publicadas en EE.UU. y Europa en el campo de estudios de los medios y la comunicación a partir de las siguientes preguntas: ¿qué significa "pluralidad de voces" cuando hablamos de revistas de alto impacto publicadas en inglés en el campo de los estudios de comunicación y medios? ¿Y en qué medida concierne dicha pluralidad a América Latina?

En base a Llorens (2002), pluralidad alude aquí a una amplia variedad de pertenencias institucionales y países de residencia entre autorxs y evaluadorxs, mientras que diversidad alude a la multiplicidad y la no uniformidad como cualidades del conjunto de artículos publicados en una revista a lo largo del tiempo, incluida la variación de perspectivas epistemológicas, temáticas y geopolíticas. Es importante notar que, a partir del aporte de Napoli referido a las políticas de comunicación, entendemos la diversidad no sólo en términos de contenidos, sino también en términos de exposición a "las diferentes perspectivas representadas por [...] fuentes antagónicas" (1999, p. 250). De este modo, el pluralismo, en cuanto suma de pluralidad y diversidad, refiere no sólo a las instancias de producción de contribuciones latinoamericanas a las publicaciones académicas globales dedicadas a la comunicación, sino también, a los niveles de distribución, exhibición y acceso a esos puntos de vista. 


\section{Marco metodológico}

\subsection{Intervenir concretamente en el estado de situación: de estudio cualitativo a edición especial}

\subsubsection{El estudio cualitativo}

A partir de la intención expresa de la editora responsable de CT de alentar a "autores y editores a poner de relieve los contextos históricos, culturales y políticos en los que los enfoques teóricos se definen" (Wilkins, 2016, p. 105), nos dispusimos a investigar el archivo en línea de la revista con esta pregunta como eje: ¿qué presencia ha tenido la producción teórica latinoamericana en la revista desde comienzos de la década de 1990? Para responderla, realizamos una búsqueda utilizando a la región y sus países como palabras clave. Dicha estrategia nos reportó un total de ocho artículos publicados entre 1992 y febrero de 2016 que hacen referencias sustantivas a América Latina (Block, 2013; Ceisel, 2011; Davis, 2015; Lozano, 1992; Rodríguez, 2001; Scholz, 2016; Sypher, McKinley, Ventsam, \& Valdeavellano, 2002; Vásquez \& Cooren, 2013).

El análisis cualitativo de esos ocho artículos nos permite afirmar que, si bien América Latina apenas está presente en la revista, esa presencia harto limitada es, tomada en su conjunto, multidimensional. América Latina es abordada como un campo de prácticas culturales y de investigación que aporta elementos al análisis interregional; como un emplazamiento para la intervención y el trabajo de campo de investigadorxs extranjerxs; como un ejemplo empírico más o menos contextualizado lo arbitrario); como el punto de origen de una teoría crítica original y de una política de resistencia a las hegemonías, y como una zona de hibridación material y simbólica (Enghel \& Becerra, 2018). Ya sea como idea, ya sea como realidad, la América Latina surgida de nuestra indagación del archivo de CT puede ser leída agonísticamente, esto es, de forma tal que manifiesta "diferencias existentes y da lugar a la disputa respetuosa entre posiciones políticas claramente distanciadas" (Raeijmaekers \& Maeseele, 2015, p. 1046) $)^{5}$.

En términos de construcción teórica, tres de los ocho artículos adoptan enfoques críticos que se basan en, y contribuyen a, un cuerpo de producción de conocimientos latinoamericano y que, a la vez, dan cuenta de debates y preocupaciones que van más allá de la región. Cabe destacar que esos tres artículos fueron escritos por académicas mujeres (Rodríguez, 2001; Ceisel, 2011; Scholz, 2016), sumando una muy necesaria diversidad al canon típicamente masculino y elitista del campo disciplinar (Chaffee, Gómez-Palacio \& Rogers, 1990). La diversidad geopolítica de esta pequeña muestra es extremadamente limitada. Muy pocos latinoamericanos están representados en sus contenidos y todxs Ixs autorxs estaban afiliadxs a universidades de EE.UU., Canadá y Australia al momento de escribir los artículos. En lo que respecta al idioma de publicación de la literatura académica citada en los artículos, encontramos una variación significativa (inglés, castellano, portugués, alemán y francés), lo que en algunos casos contribuye a una hibridez teórica productiva.

\subsection{De la investigación a la acción}

Ante la evidencia surgida de nuestro estudio cualitativo, CT aceptó nuestra propuesta de realizar una edición especial. El llamado a presentar contribuciones se difundió en noviembre de 2016. La labor editorial comenzó en marzo de 2017 con la lectura de todas las contribuciones recibidas, para verificar que fueran consistentes con la propuesta temática y con los lineamientos de la revista. Nuestra primera decisión editorial fue no rechazar ninguna contribución de entrada debido a cuestiones gramaticales o sintácticas, distinguiendo así la calidad científica de la habilidad de lxs autorxs para escribir en inglés en tanto lengua no materna6 ${ }^{6}$.

A fin de garantizar una variedad de perspectivas, todas las contribuciones seleccionadas para revisión de pares fueron evaluadas por un/a especialista del Norte y un/a especialista de Latinoamérica. En los casos en que la primera ronda de revisión dio lugar a evaluaciones sumamente diferentes, solicitamos una tercera revisión. Cabe destacar que las diferencias en las evaluaciones no estuvieron claramente alineadas con las afiliaciones regionales, lo cual resultó desconcertante pero formativo ${ }^{7}$. Si bien como editorxs no descartamos ninguna de las evaluaciones recibidas, en algunos casos optamos 
por señalarles a lxs autorxs nuestro desacuerdo con ciertos comentarios de evaluadorxs que, a nuestro juicio, constituían opiniones más que observaciones sustancialmente argumentadas. Por medio de estas prácticas procuramos definir así una posición agonista respecto del proceso editorial lver Raeijmaekers \& Maeseele, 2015, pp. 10461047 para una discusión vinculada; ver también Enghel \& Becerra, 2020).

Cada uno de los cuatro artículos publicados en la edición especial (Scolari \& Rodríguez-Amat, 2018; Vásquez-Donoso, Marroquín-Velásquez \& AngelBotero, 2018; Arcila, Barranquero \& González, 2018; Zarowsky \& Justo Von Lurzer, 2018) fue sometido a una extensa y cuidadosa revisión. La edición especial incluyó, además, recensiones bibliográficas de la labor de dos académicxs latinoamericanxs cuyas contribuciones substanciales al campo no han sido traducidas al inglés: Eliseo Verón (Raimondo Anselmino, 2018) y Rossana Reguillo (Feixa \& Figueras-Maz, 2018). Dichas recensiones apuntaron a promover la valoración crítica de publicaciones disponibles solo en castellano len línea con Wilkins, 2016) y pretendieron llamar la atención de la audiencia de CT sobre la importancia crucial de la traducción como estrategia orientada a promover el pluralismo académico a nivel internacional.

\subsection{Propósitos de la acción}

Si bien los artículos publicados fueron cuidadosamente seleccionados a fin de destacar la relevancia de América Latina como realidad y como idea en el siglo XXI en los círculos académicos del Norte, "La teoría latinoamericana de la comunicación hoy" no tuvo la pretensión de representar la pluralidad y diversidad de los problemas y desafíos planteados en años recientes por académicxs de la región ni la riqueza de la resultante producción teórica. Dado el predominio de la producción académica generada en EE.UU. característico de CT a lo largo de los años (Waisbord, 2019, p. 97), concebimos la edición especial como una intervención concreta en los modos históricos de operar de la revista y, por extensión, en la más vasta escena que ICA representa en tanto es una de las dos asociaciones internacionales más grandes de investigadores de comunicación y medios ${ }^{8}$. La iniciativa apuntó a si- tuar a la teoría de la comunicación latinoamericana momentáneamente en el centro de las discusiones que tienen lugar en esos nodos de saber/poder la ponerla en el candelero), a subrayar cuánto se la ha ignorado y a llamar la atención sobre las formas limitadas y estereotípicas en que se suele entender y abordar empírica y teóricamente a América Latina. En aras de la pluralidad y la diversidad, decidimos, además, apartarnos de los reiterados homenajes al muy conocido canon de la teoría de la comunicación latinoamericana que se remonta a las décadas de 1970 y $1980^{\circ}$. En cambio, la edición especial se centró en contribuciones más recientes y menos conocidas en el Norte. El laborioso proceso editorial implicó lidiar con varias dificultades que consideramos en la siguiente sección.

\section{Resultados}

\subsection{La edición como práctica problemática: lecciones aprendidas y desafíos a futuro}

\subsubsection{Latinoamericanidad}

Cuando en 2016 investigamos el archivo de CT, buscamos no solo referencias a América Latina sino también pluralismo y diversidad en los sentidos definidos anteriormente en este artículo. En este sentido, nuestra aproximación no fue predeterminada, sino abierta. Nos guiamos por la pregunta planteada por el historiador Hugo Chumbita a fines de la década de 1980: si Latinoamérica existe, "¿se trata de una realidad o de una idea?" (Chumbita, 1987).

En tanto construcción ligada con la identidad, la latinoamericanidad viene siendo discutida en el campo de la comunicación desde hace años (Martín-Barbero, 2001). ¿De qué hablamos cuando nos referimos a los estudios de comunicación latinoamericanos? ¿Qué características distinguen a una contribución académica en términos de su latinoamericanidad? Estas son preguntas centrales, que Ixs colegas que contribuyeron con artículos a la edición especial de CT enfocaron de diferentes modos dependiendo de sus perspectivas epistemológicas.

Idealmente, las diferentes perspectivas epistemológicas respecto de esta cuestión debieran ser 
discutidas regularmente por la así llamada "comunidad académica global" a lo largo y a lo ancho de foros de investigación nacionales, regionales e internacionales como parte de un ejercicio colectivo de reflexividad (Dean, 2017). El objetivo de tales discusiones públicas sería, a nuestro modo de ver, apuntar no al consenso, sino a "reconocer la heterogeneidad social y las disputas como constitutivas de la política democrática" (Raeijmaekers \& Maeseele, 2015, p. 1047). Desde nuestra perspectiva, y tal como observa la socióloga argentina Fernanda Beigel, sólo cabe pensar a América Latina como unidad regional a partir del reconocimiento de su heterogeneidad e inequidades internas (Beigel, 2014b, p. 746). En el caso de la edición especial en cuestión, fue crucial contar con la disposición de la editora responsable de CT a correr el riesgo de llamar a la presentación de contribuciones en base a su latinoamericanidad. Se trató de una decisión arriesgada precisamente debido a las controversias existentes respecto de qué significa latinoamericanidad ( ¿se trata de una cualidad unánime y singular o en debate y plural?), de a quién le corresponde definirla y de por qué importa. Autorxs y evaluadorxs tendieron a abordar la latinoamericanidad de maneras distintas. Ello demandó grandes esfuerzos de síntesis, como editorxs, a fin de encontrarles el sentido a las evaluaciones dispares y de gestionar las disonantes reacciones de ciertxs autorxs a los comentarios de lxs evaluadorxs. Hacer lugar a la heterogeneidad en las evaluaciones y, a la vez, garantizar que los estándares de calidad científica de CT fueran respetados, constituyó un desafío.

\subsubsection{Cuestiones disciplinares}

La tarea editorial implicó, además, afrontar cuestiones netamente disciplinares, tanto con evaluadorxs como con autorxs. ¿Qué debe considerarse teoría comunicacional y qué no? ¿Qué es novedoso y qué no lo es? ¿Cuándo una contribución es teóricamente sustantiva debido a su enfoque latinoamericano? ¿Qué hace a una contribución teóricamente sustantiva más allá de su foco latinoamericano? ¿Y a quién le corresponde decidir y por qué? En el proceso de resolver qué publicar en base a los dictámenes de Ixs evaluadorxs, nuestros esfuerzos por contribuir a la pluralidad consistieron, en parte, en negociar diferentes presiones por excluir "esto" o "aquello" en nombre de definiciones preferenciales, aunque implícitas, de qué es la "teoría de la comunicación" y, por ende, qué es "apropiado" o "impropio". Las presiones para excluir provinieron no sólo de algunxs evaluadorxs, situadxs por definición en posición de ejercer poder sobre los resultados del proceso editorial. También hubo autorxs que presionaron por hacer caso omiso de las críticas razonablemente justificadas que se les plantearon, bajo el argumento de que sabían más acerca de la latinoamericanidad que un revisor "otro" apresuradamente tipificado como incapaz de entender lo que habían querido decir. La tarea editorial nos permitió confirmar desde la práctica que la noción misma de teoría es puesta en cuestión cuando diferentes culturas académicas entran en diálogo. En ese escenario, la tensión surgida de la diferencia habrá de ser considerada enriquecedora desde una perspectiva agonista lun estímulo para el "debate público y esencialmente político", para volver a Raeijmaekers \& Maeseele, 2015, p. 1047), percibida como un problema a ser resuelto por quienes abogan por el consenso unilateral o, bien, rechazada como inaceptable por lxs defensorxs del separatismo. Como editores procuramos hacer lugar al agonismo mientras negociábamos resoluciones. En este sentido, la tarea de producir la edición especial de CT atendiendo a perspectivas múltiples implicó interpretar los pedidos a los autorxs y las reacciones a las evaluaciones de lxs revisorxs simultáneamente desde distintos puntos de vista.

\subsubsection{Contacto entre Norte y Sur}

Las presiones para excluir ciertas formas de concebir la teoría constituyeron, además, un síntoma de la tercera cuestión sobre la que queremos reflexionar: el escaso contacto entre las "comunidades" (si empleamos un término más bien esperanzador) - "tribus" (si utilizamos un término más crítico) académicas del Norte y del Sur. Ese escaso contacto se manifestó como cierta torpeza en el trato mutuo, evidente a pesar de que dicho trato tuvo lugar mediado por la distancia (Silverstone, 1999) y el anonimato propixs del proceso de revisión de pares. Existen, desde luego, causas materiales bien conocidas para la falta de contacto. Ejemplos de esas causas materiales son la supremacía del inglés en las ciencias sociales, las diferencias de lenguaje que obstaculizan la comunicación frecuente y fluida, y los escasos recursos disponibles en América Latina para acceder a las publicaciones mejor indizadas, para viajar a grandes conferencias internacionales u organizar seminarios internacionales en la región, necesarios a fin de encontrarse 
cara a cara y socializar $(0 r t i z, 2009)^{10}$. Dados estos impedimentos, lxs académicxs de la región se encuentran, de hecho, en una posición de desventaja estructural.

Pero los malestares detectados fueron más allá de dichos obstáculos materiales lque la edición especial apuntó a superar) y constituyeron tanto la causa como la consecuencia de una distancia entre colegas de ambos "lados" - Norte y Sur - expresada como crítica mutua, tal que la responsabilidad por lo irresuelto o no compartido se le atribuía al "otro". Este tipo de crítica tuvo una presencia significativa aunque no fue predominante ${ }^{11}$, y se manifestó de maneras estereotipadas: como ideas fijas acerca de cómo eran o debían ser los artículos y sobre quién era responsable cuando esos requisitos no se cumplían. Actitudes de ese tipo, si no se las advierte y no se reflexiona al respecto, tienden a reforzar el statu quo de distancia y exclusión y, por ende, las partes involucradas -editorxes, autorxs, evaluadorxs - deben prestar especial atención a la micropolítica del pluralismo académico. Además, quienes nos reconocemos como provenientes del Sur pero estamos afiliadxs a universidades del Norte tenemos la posibilidad y, por lo tanto, la responsabilidad, no de actuar como "policía fronteriza", sino de dar el ejemplo cruzando puentes e invitando al diálogo y el debate entre ambas partes a partir de nuestras propias experiencias de hablar a "ambos lados"12. Al mismo tiempo, cabe prestar atención a los prejuicios no solo de lxs académicxs del Norte respecto de sus colegas del Sur, sino también a la dinámica inversa. La experiencia de producir la edición especial de CT puso en evidencia que, lejos de ser exclusivamente neocolonial, el prejuicio puede circular en ambas direcciones.

\section{Conclusiones}

\section{1 ¿Cómo contribuir al pluralismo internacional en los estudios de medios y comunicación desde América Latina? Limitaciones y posibilidades}

Habiendo discutido la importancia de las disposiciones y actitudes personales para iniciativas de pequeña escala como la analizada aquí y, por lo tanto, la importancia del accionar individual o micropolítico, queremos enfatizar la importancia de abordar también el problema desde una perspectiva estructural. Nos interesa particularmente considerar los límites y las posibilidades para el pluralismo académico y la internacionalización del campo en lo que respecta a América Latina. Volviendo a las observaciones de Sonia Livingstone (2007, p. 275) sobre las variedades existentes de proyectos de internacionalización, pensamos que la responsabilidad por "desoccidentalizar" y diversificar los estudios de medios y comunicación no debería recaer principalmente sobre las regiones o países excluidos de nodos de saber/poder tales como la ICA y sus publicaciones: la inequidad existe y no debe ser ignorada por quienes la promueven o se benefician directa o indirectamente con ella. Pero prestar atención a la dimensión estructural significa tener en cuenta también las limitaciones provenientes de la propia América Latina. Si las ignoráramos, estaríamos contribuyendo, por omisión, a coproducir un pluralismo limitado y una internacionalización desigual.

Existen tres tipos de limitaciones estructurales que nos importa destacar aquí.

Primero, que el contexto general en el que se producen los estudios de comunicación en América Latina se caracteriza, por un lado, por la escasez de sistemas de medios de servicio público sin fines comerciales encargados de proporcionar contenido audiovisual plural, diverso y de alta calidad ${ }^{13} \mathrm{y}$, por otro, por la hiperconcentración de la propiedad de los medios privados. En este entorno, las instituciones estatales tienden a actuar como defensoras y clientes de los medios privados la través de políticas y de pautas publicitarias, respectivamente) en lugar de ser garantes de la igualdad y, por ende, las iniciativas para promover el pluralismo se limitan a los esfuerzos de la sociedad civil y las organizaciones comunitarias (Fox \& Waisbord, 2002; Hallin \& Papathanassopoulos, 2002; Rincón, 2001; De Mateo \& Bergés, 2009; Becerra \& Mastrini, 2017). Este contexto es performativo de los debates públicos y la debilidad relativa de espacios sociales que cultiven el pluralismo permea, podría decirse gramscianamente, en las condiciones generales en las que lxs académicxs latinoamericanxs producimos ideas y propuestas. A diferencia de la estructuración del espacio público en países europeos y en ciertos sectores y regiones de EE. UU. y Cana- 
dá, en América Latina el sentido de lo público es constantemente disputado, se halla mayormente mercantilizado por el tipo de conformación de sus sistemas de producción y circulación de noticias, opiniones e ideas masivas, y ha sido y es inestable. En este "continente" se desarrolla una producción intelectual de características distintivas.

Segundo, que los sistemas académicos latinoamericanos operan mayoritariamente en ausencia de políticas públicas y recursos - materiales y simbólicos- destinados a fomentar la amplia difusión del conocimiento producido a escala nacional, regional e internacional.

Tercero, que la presión institucional a fin de que los estudios de comunicación y medios latinoamericanos adopten preocupaciones temáticas y formatos de publicación típicos del trabajo académico en el Norte erosiona la pluralidad y la diversidad para Sur y Norte por igual. Nuestra hipótesis es que el segundo y el tercer tipo de limitación estructural que aquí señalamos fomentan el pluralismo asimétrico en toda la región, de modo tal que ciertos académicos latinoamericanos están sobrerrepresentados en las publicaciones internacionales más prestigiosas mientras que otros apenas figuran, "en parte como resultado de la distribución desigual del capital cultural y lingüístico entre los científicos, en parte debido a restricciones estructurales" $y$, además, como producto de trayectorias diferenciales de profesionalización (Beigel, 2014a, p. 622).

A fin de que las contribuciones latinoamericanas al pluralismo internacional en los estudios de medios y comunicación se tornen significativas y sostenibles, es esencial que lxs académicxs unamos fuerzas con el propósito de democratizar sus condiciones de posibilidad dentro de los sistemas académicos nacionales y los grupos regionales de interés (tales como las asociaciones de investigadores) en vez de limitarnos a responsabilizar a los actores dominantes del Norte por su perdurable posición de privilegio. Esperamos que los argumentos planteados tanto en este artículo como en la edición especial a la que se refiere alienten debates y acuerdos estratégicos respecto de cómo avanzar en esta dirección y contribuyan, así, a fomentar una redefinición de la capacidad de acción colectiva fundada en la comprensión de las limitaciones estructurales a enfrentar.

\section{2 ¿Cuál es el futuro?}

"La teoría latinoamericana de la comunicación hoy: cartografía de los desarrollos contemporáneos y su relevancia global" se publicó en mayo de 2018, con acceso abierto ${ }^{14}$ durante el primer mes posterior a su edición. Lxs autorxs publicadxs se sumaron a la tarea de comunicar la edición especial como esfuerzo colaborativo en vez de promover sus respectivos artículos como una cuestión de logro individual. Las reflexiones inmediatas derivadas del proceso fueron discutidas en una preconferencia de ICA en 2018 (Enghel \& Becerra, 2020). Al proponer este artículo como complemento, esperamos provocar la discusión agonista del papel de América Latina en términos de contribuir al pluralismo académico en el contexto de un campo internacionalmente desigual (Enghel \& Becerra, 2018). Más allá del argumento según el cual la región merece un espacio en las revistas académicas de alto impacto como garantía de inclusión en un campo desigual, consideramos que la pluralidad y la diversidad de incluir puntos de vista latinoamericanos entre otras posturas no occidentales generaría y contribuiría a un debate más vigoroso y mejor informado de los desafíos globales que requieren atención urgente. Dicha pluralidad y diversidad no se producirán como resultado de una repentina toma de conciencia por parte de los círculos académicos del Norte acerca de su poder diferencial y sus prácticas excluyentes. Materializarlas requerirá que sean coproducidas activamente por un cuerpo de académicos a ambos lados de la división Norte-Sur que cooperen sistemática y reflexivamente para modificar el statu quo.

\section{Notas}

1. Todas las citas directas correspondientes a textos publicados originalmente en inglés han sido traducidas al castellano por lxs autorxs.

2. Al momento de escribir este artículo, no tenemos conocimiento de la existencia de estudios cualitativos que investiguen la pluralidad temática en las revistas de ICA desde una perspectiva geopolítica.

3. La cuestión de si un club selecto de países tan pequeño debiera considerarse 'global' o 'globalizado' merece ser discutida. No lo hacemos aquí porque dicha discusión excede los propósitos y posibilidades de este artículo. 
4. Originalmente desarrollada en la tesis de Llorens sobre la concentración estructural del sistema de medios de comunicación y las políticas públicas al respecto.

5. Esta caracterización de una posición agonística que Raeijmaekersand y Maeseele (2015) proponen en su análisis de los vínculos entre medios, pluralismo y democracia se basa en la noción de Chantal Mouffe de "agonismo pluralista". Mouffe (2005) contempla a la política como un conflicto entre posiciones contrarias que no apuntan a eliminarse unas a otras sino que pueden, en última instancia, respetar su derecho a coexistir. En base a la experiencia que revisamos críticamente aquí, creemos que una política editorial agonista contribuiría a dar un carácter internacional plural a la literatura académica publicada en inglés sobre medios y comunicación.

6. Las cuestiones idiomáticas, donde las hubo, se resolvieron en un estadio más avanzado del proceso de edición.

7. Curiosamente, la decisión de otorgarles representación a todas las partes al convocar a lxs evaluadorxs, a la manera de los foros de Naciones Unidas, internacional, no se tradujo en alineamientos geopolíticos.
8. La otra es la Asociación Internacional de Estudios en Comunicación Social, AIERI (IAMCR por su sigla en inglés).

9. Para una discusión de este problema, ver Enghel \& Becerra (2018, p. 120-121).

10. Queda abierta la pregunta respecto del potencial impacto del pasaje de las conferencias internacionales a formatos virtuales durante la pandemia de COVID-19 sobre el problema de la falta de oportunidades para socializar entre Sur y Norte.

11. La mayoría de lxs evaluadorxs y autorxs demostraron enorme sensibilidad y curiosidad por un "otro" epistemológicamente diferente.

12. Si bien lxs autorxs tenemos un origen nacional común, nuestras trayectorias académicas se han desarrollado en América Latina y Europa del Norte, respectivamente.

13. Distinto es el caso en EE. UU., donde existe un sistema de medios de servicio público cuasi-universal, aunque recibe menos financiamiento y es menos popular que los medios públicos en Europa. Ver https://www.pbs.org/about/about-pbs/ [link chequeado el 11 de noviembre de 2020]

14. La revista pertenece a ICA, pero es administrada comercialmente por Oxford University Press.

\section{Referencias}

Arcila, C., Barranquero, A. \& González, E.(2018). From Media to Buen Vivir: Latin American Approaches to Indigenous Communication. Communication Theory, 28 (2), 180-201.

Becerra, M. \& Mastrini, G. (2017). La concentración infocomunicacional en América Latina (2000-2015): nuevos medios y tecnologías, menos actores. Bernal: Universidad Nacional de Quilmes.

Beigel, F. (2014a). Introduction: Current tensions and trends in the World Scientific System. Current Sociology, 62(5), 617-625.

Beigel, F. (2014b). Publishing from the periphery: Structural heterogeneity and segmented circuits. The evaluation of scientific publications for tenure in Argentina's CONICET". Current Sociology, 62(5), 743-765.

Block, E. (2013). A culturalist approach to the concept of the mediatization of politics: The age of "Media Hegemony." Communication Theory, 23(3), 259-278. https://doi. org/10.1111/comt.12016 
Ceisel, C. M. (2011). El rock star perfecto? Theorizing Juanes and new directions in crossover celebrity. Communication Theory, 21(4), 413-435. https://doi.org/10.1111/ j.1468-2885.2011.01393.x

Chaffee, S. H., Gomez-Palacio, D. C., \& Rogers, D. E. M. (1990). Mass communication research in Latin America: Views from here and there. Journalism Quarterly, 67(4), 1015-1024.

Chumbita, H. (1987). Latinoamericanidad: la apertura a lo imaginario. Alternativa Latinoamericana, 6 .

Curran, J. \& Park, M.J.(2000). 'Beyond Globalization Theory'. En J. Curran \& M.J. Park (eds) De-Westernizing Media Studies. London: Routledge.

Davis, S. (2015). Citizens' media in the Favelas: Finding a place for community-based digital media production in social change processes. Communication Theory, 25(2), 230-243. https://doi.org/10.1111/comt.12069

Dean, J. (2017). Doing reflexivity: An introduction. Bristol: Policy Press and Bristol University Press.

De Mateo, R. \& Bergès, L. (2009). Los retos de las televisiones públicas: financiación, servicio público y libre mercado. Sevilla: Comunicación Social.

Doyle, G. (1997). From Pluralism to ownership. Europe's emergent policy on media concentrations navigates the doldrums. Journal of Information, Law and Technology, 3. https://warwick.ac.uk/fac/soc/law/elj/jilt/1997_3/doyle/

Enghel, F. \& Becerra, M. (2018). Here and There: (Re)Situating Latin America in International Communication Theory. Communication Theory, 28 (2), 111-130, https:// doi.org/10.1093/ct/qty005

Enghel, F. \& Becerra, M. (2020). How to incorporate Latin American communication studies into northern/western Circles? En Orchard, X., Santamaria, S.G., Brambila, J. \& Lugo-Ocando, J. (eds.) Media and governance in Latin America: Toward a plurality of voices. New York: Peter Lang Publishing Group, pp. 59-73.

Feixa, C. \& Figuera-Maz, M. (2018). Emergence of (Hybrid) Youth Cultures. Communication Theory, 28 (2), 224-228.

Fox, E., \& Waisbord, S. (Eds.) (2002). Latin politics, global media. Austin, TX: University of Texas Press.

Ganter, S. \& Ortega, F. (2019). The Invisibility of Latin American Scholarship in European Media and Communication Studies: Challenges and Opportunities of De-Westernization and Academic Cosmopolitanism. International Journal of Communication, 13, 68-91.

Hallin, D. C., \& Papathanassopoulos, S. (2002). Political clientelism and the media: Southern Europe and Latin America in comparative perspective. Media, Culture \& Society, 24(2), 175-195.

Livingstone, S. (2007). Internationalizing media and communication studies: Reflections on the International Communication Association. Global Media and Communication, 3(3), 273-288. 
Llorens, C. (2002). Concentración de empresas de comunicación y el pluralismo: la acción de la Unión Europea, Tesis Doctoral defendida en la Universidad Autónoma de Barcelona, Barcelona.

Lozano, E. (1992). The force of myth on popular narratives: The case of melodramatic serials. Communication Theory, 2(3), 207-220. https://doi.org/10.1111/j.1468-2885.1992. tb00039.x

Martín-Barbero, J. (2001) Reconfiguraciones comunicativas de lo público. Guadalajara: TESO.

Mouffe, C. (2005). On the political. London: Routledge.

Napoli, P. (1999). Deconstructing the diversity principle, Journal of Communication, Autumn, 7-34.

Ortiz, R. (2009). La supremacía del inglés en las ciencias sociales. Buenos Aires: Siglo XXI.

Raeijmaekers, D. \& Maeseele, P. (2015). Media, pluralism and democracy: what's in a name? Media, Culture \& Society, 37(7), 1042-1059.

Raimondo-Anselmino, N. (2018). A Semio-Anthropological Perspective on Mediatization: Semiosis, 2 by Eliseo Verón. Communication Theory, 28 (2), p. 229-233.

Rich, M. (1993, August 11-14). Culture and communication in Latin America. Paper presented at the 76th Annual Meeting of the Association for Education in Journalism and Mass Communication. ERIC Document Reproduction Service (EDRS) ED 361803 , Kansas City, Missouri.

Rincón, O. (Ed.). (2001). Televisión pública: Del consumidor al ciudadano. Bogotá: Convenio Andrés Bello.

Rodríguez, C. (2001). Shattering butterflies and amazons: Symbolic constructions of women in colombian development discourse. Communication Theory, 11(4), 472494. https://doi.org/10.1111/j.1468-2885.2001.tb00254.x

Scholz, T. M. (2016). Beyond "Roaring Like Lions": Comadrismo, counternarratives, and the construction of a Latin American Transnational subjectivity of feminism. Communication Theory, 26(1), 82-101. https://doi.org/10.1111/comt.12059

Scolari, C. \& Rodríguez-Amat, J. (2018). A Latin American Approach to Mediatization: Specificities and Contributions to a Global Discussion About How the Media Shape Contemporary Societies. Communication Theory, 28 (2), 131-154.

Silverstone, R. (1999). Why Study the Media? London: SAGE.

Sypher, B. D., McKinley, M., Ventsam, S. \& Valdeavellano, E. E. (2002). Fostering reproductive health through entertainment-education in the Peruvian Amazon: The social construction of Bienvenida Salud. Communication Theory, 12(2), 192-205. https://doi.org/10.1111/j.1468-2885.2002.tb00266.x

Vásquez, C. \& Cooren, F. (2013). Spacing practices: The communicative configuration of organizing through space-times. Communication Theory, 23(1), 25-47. https:// doi.org/10.1111/comt.12003

Vásquez-Donoso, C., Marroquín-Velasquez, L. \& Angel-Botero, A. (2018). In Search of a Latin American Approach to Organizational Communication: A Critical Review of Scholarship (2010-2014). Communication Theory, 28 (2), 155-179. 
Waisbord, S. (2016). Communication studies without frontiers? Translation and cosmopolitanism across academic cultures. International Journal of Communication, 10(2016), 868-886.

Waisbord, S. (2019) Communication: a post-discipline. Cambridge: Polity.

Wilkins, K. G. (2016). Introduction to editorship 2016. Communication Theory, 26(2), 103105. https://doi.org/10.1111/comt.12097

Zarowsky, M. \& Justo Von Lurzer, C. (2018). Communication Research in Argentina (20012015): Between Expansion and Intellectual Intervention. Communication Theory, 28 (2), 202-223.

\section{- Sobre los autores:}

Martín Becerra es Investigador Principal en el Conicet y Profesor Titular por concurso en la Universidad Nacional de Quilmes y en la Universidad de Buenos Aires. Es doctor en Ciencias de la Información (U. Autónoma de Barcelona). Especialista en políticas de medios, telecomunicaciones y TIC.

Florencia Enghel es Marie Curie Fellow 2020-2023 con financiamiento del programa Horizon 2020 de la Unión Europea y Profesora Asociada en la Universidad de Jönköping, Suecia. Es Doctora en Medios y Comunicación (Universidad de Karlstad). Especialista en comunicación para el cambio social.

\section{- ¿Cómo citar?}

Becerra, M. \& Enghel, F. (2021). Pluralismo agonista en la internacionalización de los estudios latinoamericanos de la comunicación: reflexiones a partir de la práctica. Comunicación y Medios, (43), 24-35. https://doi.org/10.5354/0719-1529.2021.60718 